\title{
Do BIM ao CIM: Contribuição ao Desenvolvimento de Projetos Urbanos $^{1}$
}

\section{From BIM to CIM: Contribution to the Development of Urban Projects}

\author{
Freire, Fábio1; \\ 'Universidade Tecnológica Federal do Paraná - UTFPR, Rua Marcílio Dias, 635 - \\ Apucarana, Paraná, Brasil, fabiofreire@utfpr.edu.br
}

\begin{abstract}
RESUMO
Atualmente, a Modelagem da Informação na Cidade (CIM) pode ser considerada uma ferramenta indispensável na área temática do planejamento urbano. Com a evolução das ferramentas de Modelagem da Informação na Construção (BIM) já é possível utilizar métodos que desenvolvem modelos paramétricos na escala da cidade, nesse contexto, para muitos autores o BIM faz parte do CIM. A apresentação de experiências de projetos urbanos, utilizando métodos de modelagem em BIM ou a partir de softwares com programação algorítmica, contribuem para que profissionais de planejamento urbano conheçam as abordagens fora do padrão, funções pouco estudadas e novos recursos dos quais muitos usuários nem sequer estão cientes. No futuro próximo, com o CIM, será possível armazenar todas as informações de uma cidade e visualizar as relações entre os edifícios, infraestrutura, ambiente e como elas estão se inter-relacionando.
\end{abstract}

Palavras-chave: CIM, BIM, projeto urbano.

\begin{abstract}
Currently, the Modeling of Information in the City (CIM) can be considered an indispensable tool in the thematic area of urban planning. With the evolution of the Information Modeling in Construction (BIM) tools it is already possible to use methods that develop parametric models in the city scale, in this context, for many authors BIM is part of the CIM. The presentation of urban design experiences, using BIM modeling methods or algorithm programming software, helps urban planning professionals to know non-standard approaches, little-studied functions, and new features of which many users are not even aware. In the near future, with the CIM, it will be possible to store all the information of a city and to visualize the relationships between buildings, infrastructure, environment and how they are interrelating.
\end{abstract}

Keywords: CIM, BIM, urban project.

${ }^{1}$ FREIRE, Fábio. Do BIM ao CIM: Contribuição ao Desenvolvimento de Projetos Urbanos. In: II SIMPÓsIO NACIONAL DE GESTÃO E ENGENHARIA URBANA: SINGEURB, 2019, São Paulo. Anais... Porto Alegre: ANTAC, 2019. 


\section{INTRODUÇÃO}

Uma abordagem importante para o desenvolvimento de projetos urbanos é ter uma boa sistematização de informações, principalmente para que elas sejam continuamente atualizadas, precisas e disponíveis, para todos os envolvidos no fluxo de trabalho do projeto. Atualmente, se discute intensamente como utilizar ferramentas digitais inovadoras na indústria da construção civil, com maior visibilidade para a Modelagem da Informação na Construção (BIM), porém a escala de abordagem se restringe na maioria das vezes ao edifício.

Surge então um outro conceito para tratar o BIM na escala da cidade, a Modelagem da Informação na Cidade (CIM). Alguns autores partilham da percepção que o BIM faz parte do CIM, basicamente uma extensão aplicada a bairros ou cidades inteiras (SILVA, J. F. et al. 2017). Nessa perspectiva podemos considerar que o BIM contribui para uma melhor inter-relação entre as diversas informações do edifício, então o CIM pode integrar diferentes infraestruturas e serviços de uma cidade (KHEMLANI, 2005).

É importante compreender que o CIM não deve se restringir apenas à concepção do projeto urbano, mas também, como o BIM, a todo o "ciclo de vida" da cidade ou dos sistemas urbanos (AMORIM, 2015). De acordo com Beirão, Mendes e Celani (2015) o CIM pretende integrar numa única plataforma o projeto urbano e métodos analíticos, fazendo uso das informações associadas por georreferenciamento.

Os estudos de caso, apresentados neste artigo, contém informações sobre como o CIM deriva da gestão de informações do BIM e também apresenta uma breve descrição da forma adotada no desenvolvimento dos modelos propostos. A análise realizada inclui noções de funcionamento dos modelos e como eles respondem a programação algorítmica, com o software Rhino-Grasshopper, e a utilização do método "Perfis Complexos" do software ARCHICAD.

\section{OBJETIVOS}

Apresentar experiências na utilização de métodos de modelagem BIM e programação algorítmica, aplicados a escala da cidade, compartilhando conhecimento acerca dos recursos tecnológicos e métodos disponíveis aos profissionais de planejamento urbano.

\section{METODOLOGIA}

Os estudos de caso apresentados nesse artigo fazem parte das atividades de ensino desenvolvidas no Departamento de Planejamento Urbano do Instituto de Arquitetura de Moscou (GRAPHISOFT, 2019). O método proposto aos estudantes, para construir um modelo paramétrico de projeto urbano (um dos inúmeros métodos incluídos na abordagem integrada para trabalhar na escala urbana), torna possível realizar numerosas análises de projeto simultaneamente, economizando tempo para outras tarefas importantes, como verificar dimensões, altura, silhueta e composição em 3D.

\subsection{Estudo 1 - Método de Perfis Complexos software ARCHICAD}

O método de "Perfis Complexos" permite que rapidamente e simultaneamente, em todas as projeções (seções, planos, modelos 3D), seja possível alterar a configuração de blocos, preenchendo os edifícios e suas fachadas, bem como verificando a conformidade com os padrões de insolação, ventilação e parâmetros urbanísticos, por exemplo.

O método foi utilizado, nesse caso, empregado a ferramenta "parede personalizada", para criar blocos de construção, estrutura de vias e passagens. No perfil do edifício ou em uma seção predeterminada é possível levar em consideração: o número de pavimentos, tipo de uso, densidade de ocupação, entre outros aspectos. Todos esses parâmetros podem ser alterados no processo de design, mas é necessário começar com os perfis de construção 
típicos, nomeados de acordo com o tipo de seção e o número de pavimentos.

Depois de elaborar o modelo foi possível definir todas as projeções e visualizações necessárias, para realizar cálculos básicos da legislação urbanística, verificação de insolação e ventilação. A proposta do projeto urbano, na escala de bairro, utiliza o método de "Perfis Complexos" para simular diferentes possibilidades urbanísticas (ver Figura 1).

Figura 1 - Estágio preliminar (esquerda) e etapa final - após modelagem BIM da proposta de intervenção e alterações no perfil (direita)
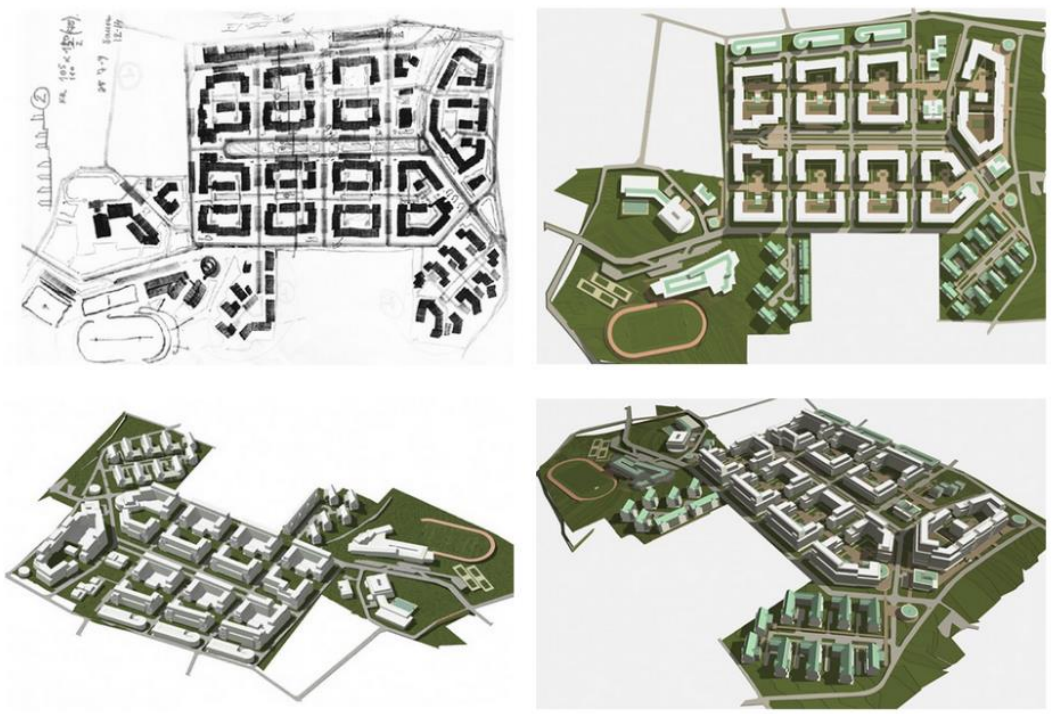

Fonte: GRAPHISOFT, 2019.

Exemplo de interface do método "Perfis Complexos" (ver Figura 2): na aba expandida da ferramenta aparece a janela com uma seção transversal do perfil e os principais parâmetros do edifício são refletidos - número de pavimentos, uso por pavimento, largura e altura do edifício, coberturas. Na janela adicional para ajuste de perfil, é possível definir o material da superfície (fachada, lajes, área local, entre outros).

Figura 2 - Estágio preliminar (esquerda) e etapa final - após modelagem BIM da proposta de intervenção e alterações no perfil (direita)
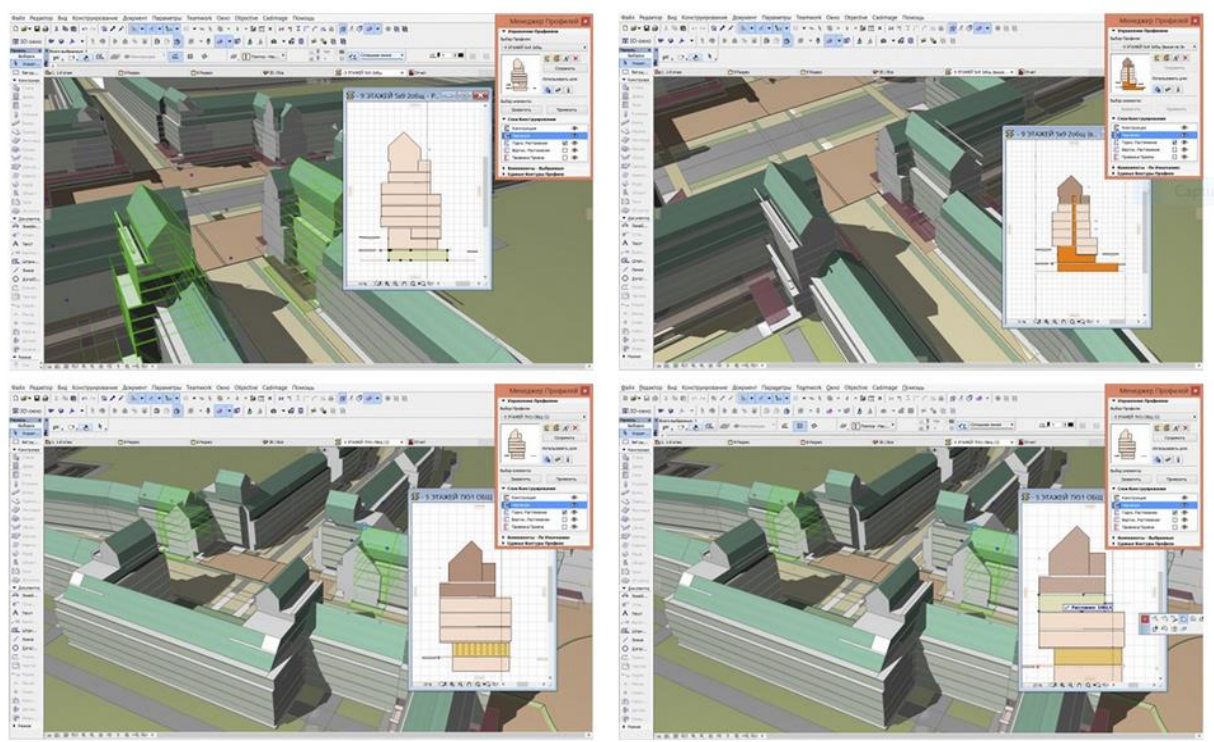

Fonte: GRAPHISOFT, 2019. 


\subsection{Estudo 2 - Método com Programação Algorítmica software Rhino-Grasshopper}

O objetivo deste estudo foi desenvolver um módulo de programação de software para a construção 3D de um fragmento do tecido urbano. O algoritmo foi escrito usando o editor de programação visual do Grasshopper, integrado ao software Rhinoceros.

O edifício foi elaborado por um código de programa, que permite simular a solução volumeespacial usando os dados de entrada dos eixos de rua e suas interseções, levando em conta muitos parâmetros (por exemplo: forma, altura do edifício, densidade, a presença de uma função social, silhueta, entre outros).

O algoritmo foi construído, considerando que é possível criar edifícios atendendo aos requisitos de um ambiente urbano "confortável", a partir de geometria simples com base nas linhas de eixo das vias (ver Figura 3).

Figura 3 - Definição do traçado inicial (acima) e proposta volumétrica de edifícios após modelagem algorítmica (abaixo)

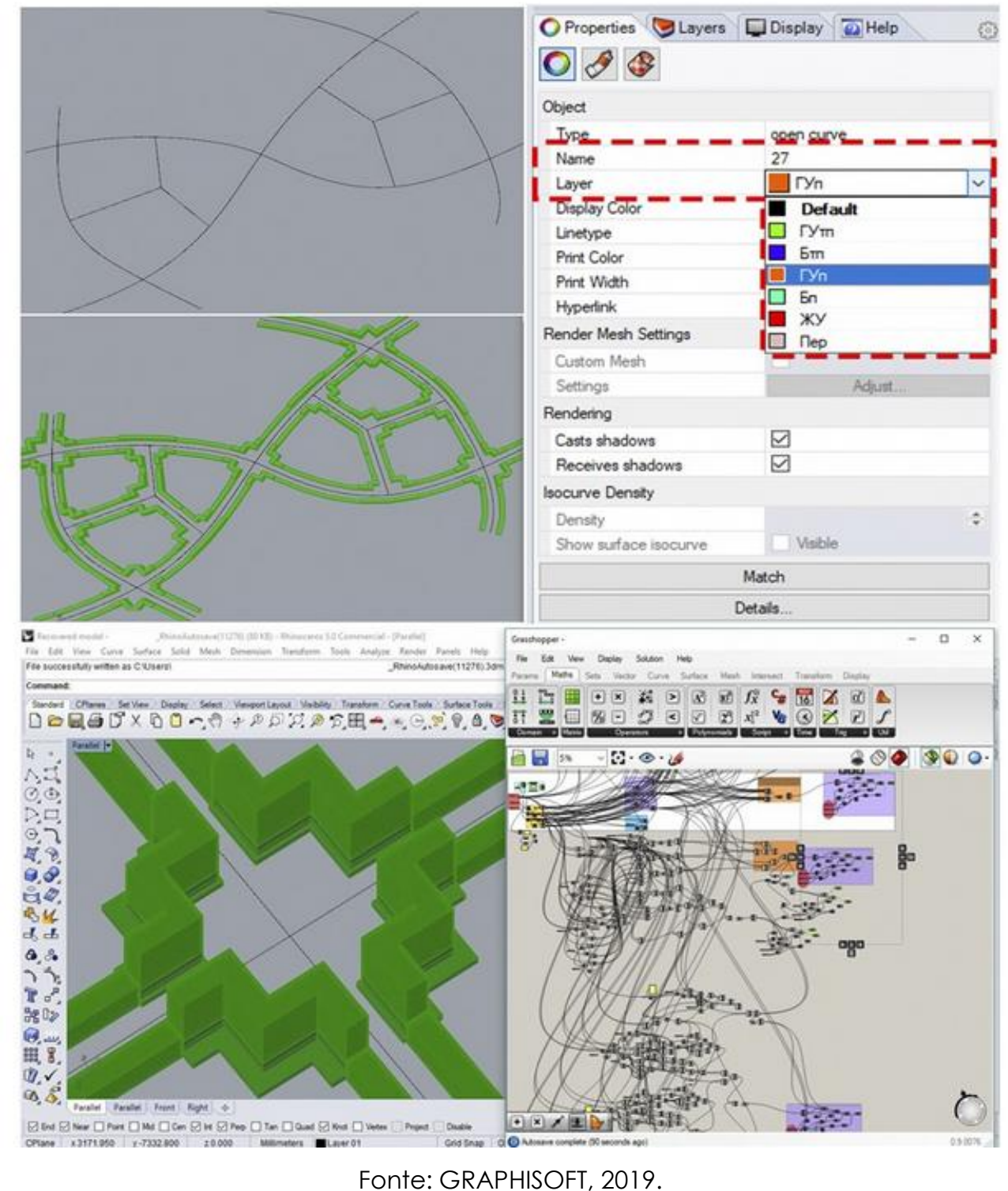

Na etapa subsequente, de desenvolvimento do algoritmo, foram introduzidos parâmetros com capacidade de analisar dados digitais. Em um primeiro momento, os parâmetros da área e densidade de edifícios residenciais. Nesse caso foi possível obter dados específicos sobre a relação uso do solo e densidade. 


\section{ANÁLISE DE RESULTADOS}

O método "Perfis Complexos" do software ARCHICAD, apresentado no estudo 1, possibilita esclarecer e/ou checar os parâmetros de altura, insolação, disposição das áreas funcionais (residencial, comercial, mista e pública), podendo ter os parâmetros rapidamente modificados, gerando novas visualizações e informações. É importante destacar que a inclusão da possibilidade de parametrização do método "Perfis Complexos" se deu a partir da versão 22 do software ARCHICAD, facilitando a criação de inúmeras possibilidades de perfis sem que fosse necessário criá-los individualmente, como verificado em versões anteriores do software.

No Estudo 2, a principal característica do algoritmo desenvolvido é a capacidade de evoluir continuamente: pode ser repetidamente incrementado, adicionando novas funções, bem como introduzindo condições adicionais que serão levadas em conta ao construir um edifício. Depois que a estrutura e a maior parte do edifício são geradas, o modelo pode ser descarregado no ambiente ARCHICAD e utilizado como um arquivo de tarefas básicas para interpretação adicional pelo projetista.

Os estudos apresentados fornecem muitas informações sobre a capacidade do processo BIM no suporte ao desenvolvimento de projetos urbanos. Todavia é necessário a integração de bancos de dados espaciais e ferramentas BIM, para a geração do projeto CIM.

\section{CONCLUSÕES}

Esse artigo reforça a importância do processo BIM em gerenciar informações na escala da cidade e principalmente, quando incorporado a outras bases de dados, auxiliar no desenvolvimento de aplicações CIM. Essas tecnologias digitais inovadoras podem ser consideradas extremamente úteis no suporte ao desenvolvimento de projetos urbanos, uma vez que são uma oportunidade de antecipar cenários e facilitar a confrontação de opções de projeto. Essa pode ser uma ferramenta útil ao intervir e/ou planejar áreas da cidade.

Na utilização de um modelo CIM é essencial desenvolver interfaces amigáveis para as várias tarefas de design, especialmente para gerenciar informações, fornecer produtos gráficos de alta qualidade e simulações tridimensionais, possibilitando dar suporte a tomada de decisões em projetos urbanos.

Diante desse contexto é importante que profissionais de planejamento urbano conheçam as abordagens fora do padrão, funções pouco estudadas e novos recursos dos quais muitos usuários nem sequer estão cientes. É preciso estar confiante de que apenas um profundo conhecimento de tecnologias inovadoras, entre elas o processo BIM e CIM, poderão ajudar a revelar todo o seu valor e influenciar os resultados, a velocidade e a qualidade do trabalho dos profissionais de planejamento urbano de maneira decisiva.

\section{REFERÊNCIAS}

AMORIM, A. L Discutindo City Information Modeling (CIM) e Conceitos Correlatos. Gestão \& Tecnologia de Projetos, [s. I.], n. 2, p. 87, 2015. Disponível em: <http://www.revistas.usp.br/gestaodeprojetos/article/view/103163>. Acesso em: 21 abr. 2019.

BEIRÃO, J. N.; MENDES, L. T.; CELANI, G. O uso do CIM (City Information Modeling) para geração de implantação em conjuntos de habitação de interesse social: uma experiência de ensino. Gestão e Tecnologia de Projetos, São Paulo, v. 10, n. 2, p. 101-112, jul./dez. 2015

GRAPHISOFT. Método expresso para construção de um modelo paramétrico de desenvolvimento urbano no ambiente ARCHICAD. Disponível em: <https://www.graphisoft.ru/users/case_studies/ac_parametric_urban_planning.html>. Acesso em: 19 abr. 2019. 
KHEMLANI, L. Around the World with BIM. Analysis, Research and Review of AEC Technology. 2013.

SILVA, J. F. et al. Análise conceitual do Building Information Modelling-BiM e City Information Modelling-CiM e contribuições na construção das cidades sustentáveis. [s. I.], 2017.

Disponível em:

<https://www.researchgate.net/publication/318801926_Analise_conceitual_do_Building_Infor mation_Modelling-BiM_e_City_Information_Modelling-

CiM_e_contribuicoes_na_construcao_das_cidades_sustentaveis>. Acesso em: 21 abr. 2019. 